DOI: https://doi.org/10.46296/gt.v5i9edespmar.0051

\title{
NIVELES DE DEPRESIÓN EN PERSONAS CON TRABAJO REMUNERADO Y DESEMPLEADAS DURANTE LA CRISIS SANITARIA DEL SARS-COV-2
}

\section{VALIDATION OF DEPRESSION LEVELS OF PEOPLE WITH PAID WORK AND UNEMPLOYED PEOPLE DURING THE SARS-COV-2 HEALTH CRISIS}

\author{
Arredondo-Aldana Karina Stephanie ${ }^{1 *}$; Gutiérrez-Izquierdo María Alexandra ${ }^{2}$ \\ ${ }^{1}$ Estudiante de la Maestría Académica con Trayectoria de Investigación en Psicología, Mención \\ Psicoterapia. Instituto de Posgrado de la Universidad Técnica de Manabí. Portoviejo, Ecuador. \\ Correo: karredondo003@utm.edu.ec. ORCID ID: https://orcid.org/0000-0002-9874-6657 \\ ${ }^{2}$ Magister en Psicología Laboral con Mención en Desarrollo Humano y de la Organización, \\ Psicóloga Industrial, Universidad Técnica de Manabí. Portoviejo, Ecuador. Correo: \\ ma.alexandragutierrezizquierdo@gmail.com. ORCID ID: https://orcid.org/0000-0003-2282-8549
}

\section{Resumen}

El año 2020 estuvo marcado por la pandemia del COVID-19 y a raíz de esto se considera la depresión como un problema recurrente en la salud mental debido a la inestabilidad económica provocada por la falta de empleo, presentándose síntomas negativos en quien la padece. Razón por la cual se hace fundamental la intervención de profesionales de la salud para evitar que los niveles de depresión se incrementen, y evitar que la población pueda llegar a padecer trastornos más complejos. El presente estudio tiene por objetivo comparar los niveles de depresión entre las personas con trabajo remunerado y las personas desempleadas en la Ciudad de Manta, durante la crisis sanitaria del SARS-CoV-2. El método utilizado en la presente investigación es el análisis estadístico descriptivo, identificando los niveles de depresión que tienen los participantes durante la crisis sanitaria del SARS-CoV-2, considerando que para este estudio se utilizó el inventario de depresión de Beck, para la detección de los niveles de depresión, el cual se fundamentó en un estudio de campo realizado de manera virtual, a través de una encuesta aplicada a 74 personas (empleados y desempleados). Obteniendo como resultados, personas con leve perturbación del estado del ánimo, depresión moderada y una depresión intermitente, se concluye que este estudio fue factible aplicando el análisis descriptivo a los datos obtenidos del inventario de depresión de Beck y determinándose que los niveles de estrés han hecho vulnerable a las personas empleadas por sobre las personas desempleadas a desarrollar una depresión.

Palabras clave: Depresión, empleo, desempleo, SARS-CoV-2.

\section{Abstract}

The year 2020 was marked by the COVID-19 pandemic, based on this, depression is considered a recurring problem in mental health due to the economic instability caused by this lack of employment, presenting negative symptoms in those who suffer from it. Therefore, the intervention of health professionals is essential to prevent the levels of depression from increasing, and to prevent the population from suffering from more complex disorders. The present study aims to compare the levels of depression between people with paid work and unemployed people in the City of Manta, during the SARS-CoV-2 health crisis. The method used to validate the research is the descriptive statistical analysis, identifying the levels of depression that the participants have during the SARS-CoV-2 health crisis, considering that for this study the Beck depression inventory was used for detection of depression levels, which was based on a virtual field study, through a survey applied to 74 people (employed and unemployed). Obtaining

Información del manuscrito:

Fecha de recepción: 06 de enero de 2022.

Fecha de aceptación: 24 de febrero de 2022.

Fecha de publicación: 04 de marzo de 2022 
as results, people with mild disturbance of mood, moderate depression, and intermittent depression. It is concluded that this study was feasible by applying the descriptive analysis to the data obtained from the Beck depression inventory and determining that stress levels have made employed people vulnerable over unemployed people when developing depression.

Keywords: Depression, job, unemployment, SARS-CoV-2.

\section{INTRODUCCIÓN}

El año 2020 se vio marcado por la pandemia del COVID-19, que según la Organización Mundial de la Salud (2019), es una enfermedad infecciosa provocada por el virus del SARS-CoV-2. Una de las consecuencias de este virus en la población que se contagia, es la presencia de cuadros respiratorios leves a moderados, los cuales se pueden agravar y provocar la muerte. Una de las maneras de contagiarse es a través de la tos 0 con un simple estornudo, incluso al hablar. Por lo que considerando a los autores Macavilca-cruz, Wuhan, \& Wuhan (2021) Domínguez, Zelaya, Gutiérrez, \& Castellanos (2020), al ser declarado pandemia se deben seguir medidas de bioseguridad e higiene, dentro de ella la cuarentena, para evitar contagios masivos y que los hospitales se llenen dejándolos incapacitados de atender demasiados casos a la vez.
La Organización Internacional del Trabajo (OIT) (2020) menciona que la crisis provocada por la pandemia del virus SARS-CoV- 2 ha tenido repercusiones de manera amplia en lo referente al plano económico y al mercado laboral, tanto en la oferta como en la demanda. Su incidencia desfavorable en la producción, que en un comienzo solo perturbó a Asia, se prolongó a las cadenas de suministro de todo el mundo. Todas las empresas, grandes, medianas y pequeñas, deben enfrentar graves problemas, en particular en los sectores relacionados con la aviación, el turismo y hotelería, así también, se ha visto un grave peligro de disminución de ingresos, aumento de la insolvencia y pérdida de puestos de trabajo en determinadas esferas.

La Comisión Económica para América Latina y el Caribe (CEPAL) (2020), refiere que, a raíz de la pandemia, en la región latinoamericana, los sectores que 
podrían sufrir las mayores contracciones son el comercio, transporte, servicios empresariales y servicios sociales, que proveen el $64 \%$ del empleo formal, Además, el $53 \%$ del empleo de la región se da en actividades informales, que serán significativamente afectadas por basarse principalmente en contactos interpersonales. Esto se ha visto manifestado en los desempleos que han ocurrido en la región y que han afectado a muchas familias $y$ trabajadores.

El Ministro de Trabajo del Ecuador, Andrés Isch, mencionó a Diario el Universo (2020) que, de marzo a septiembre, en 6 meses de la pandemia, se perdieron 410.000 empleos formales en el país. Refirió también que, en los dos primeros meses de confinamiento, marzo y abril, hubo una mayor concentración en los despidos por casos fortuitos, pero a partir de esa fecha no se ha tenido mayor movimiento. Así mismo, el ministro de trabajo (2020), destacó que las denuncias por despidos se estabilizaron en los meses de julio, agosto y septiembre, y consideró que eso se debe a que la Ley Humanitaria aplicada por parte del gobierno sirvió para disminuir la conflictividad laboral.

En concordancia a lo descrito este trabajo tiene como objetivo comparar los niveles de depresión entre las personas con trabajo remunerado y las personas desempleadas en la ciudad de Manta, durante la crisis sanitaria del SARS-CoV-2. Así también conocer cuál de las dos poblaciones son las más afectadas por este problema común en referencia a la salud mental, considerando que la inestabilidad económica provocada por la falta de empleo o el estrés provocado por factores laborales estresantes podrían traer síntomas depresivos, según Ojeda- Casares \& Gerardo de Cosio (2020), lo cual podría hacer vulnerables tanto a las personas empleadas como a las desempleadas al padecer de depresión.

Este documento se divide de la siguiente manera, en la sección 1 se encuentran lo que es la pandemia ámbito laboral y pandemia depresión, la sección 2 comprende el método y trabajos relacionados, la cual describe la forma en que se ha desarrollado este estudio, así 
también se describen los participantes involucrados, instrumento aplicado y análisis estadístico. En la sección 3 muestra los resultados obtenidos a través del análisis descriptivo, los cuales se mostrarán en tablas cruzadas que representan los datos relevantes del análisis considerándose la aplicación del test con referencia al inventario de depresión de Beck y finalmente se describen las conclusiones alcanzadas en esta investigación.

\subsection{Pandemia y ámbito laboral}

En la literatura consultada Lozano Chaguay, Robledo Galeas, \& Lozano Chaguay (2020) refieren que en un reducido periodo de tiempo el Covid19 afectó todos los procesos relacionados con la producción de bienes y servicios; así como, los procesos comerciales de todos los países, lo que generó crisis económicas envueltas de indicadores de carácter social y económico. El desempleo ha sido uno de los indicadores que más resaltan a nivel mundial, llevando consigo una afectación directa al entorno familiar, puesto que además de cumplir con las medidas de confinamientos y restricciones radicales, muchas de ellas han tenido que lidiar con el desempleo, donde no podrán contar con los ingresos per cápita que percibían, generando de esta manera algunos problemas o trastornos que afectan su salud y generan conductas inapropiadas en el núcleo familiar. Entre las diversas afectaciones están principalmente las psicológicas y dentro de ella una de las que más destaca es la depresión ante la cual los autores Castellón Leal et al., (2016) y Vera, Vélez, \& Córdova (2018) manifiestan que es un importante reto para la salud pública, puesto que es una enfermedad con elevada prevalencia y afecta a diferentes esferas de la vida de una persona, tales como: el funcionamiento familiar, social y laboral de pacientes y cuidadores, cabe recalcar que esta supone la segunda causa de discapacidad a nivel mundial.

Martínez (2020) refiere que la actual crisis sanitaria nos está mostrando la desigualdad y fragilidad de los sistemas económicos, educativos y sanitarios alrededor del mundo. Dentro de las diferentes áreas afectadas una de las más golpeada han sido los mercados de trabajo, el 
sistema de relaciones laborales y la aplicación de los instrumentos básicos de Seguridad Social.

Hernández (2020) expresa que la crisis provocada por la pandemia del SARS-CoV-2, impacta sobre las actividades sociales, las interacciones y los comportamientos del ser humando en diferentes niveles. Emocionalmente, el distanciamiento social y el autoaislamiento pueden provocar diversos problemas de salud mental, dentro de los cuales se encuentran la depresión y la ansiedad.

Balluerka et al. (2020) señala que los aspectos negativos que se insinúan en los discursos de las personas que trabajan activamente, ante la declaración del estado de alarma, están afines con la incertidumbre del fututo laboral, el abatimiento por el confinamiento, la intranquilidad por la economía futura, la gran carga de trabajo, el aburrimiento por la interrupción de actividades, el cansancio por las altas jornadas laborales y las ascendentes dificultades para realizar el teletrabajo. De igual manera al expresar incertidumbre y preocupación, se refieren la a inquietud por el bienestar de los «otros», ya sean personas cercanas o con las que tienen relación directa. Las personas que teletrabajan también refieren aspectos negativos ante esta forma de trabajo, que están relacionados con la mayor carga de trabajo, malas condiciones, y algo de incomprensión que imputan a los demás.

\subsection{Pandemia y depresión}

La OMS (2017) menciona que la depresión es un trastorno mental muy habitual y puede ser identificado por la presencia de sentimientos de culpa, trastornos del sueño o del apetito, tristeza, falta de autoestima pérdida de interés o placer, sensación de cansancio y falta de concentración según (Morales, 2017).

La depresión presentada en individuos que realizan alguna actividad remunerada es un tema de importancia en el ámbito laboral, debido a que es considerada una incapacidad, y como mencionan Castellón Leal et al. (2016) en la Unión Europea los trastornos mentales representan el $10 \%$ de la población en edad de laborar quienes tienen por lo menos un 
episodio de depresión al año, donde el descanso es de más de treinta días, lo cual constituye un gasto millonario (30 millones) por incapacidad temporal. Así mismo, representa un gasto del $1 \%$ del PIB de esta región.

De esta manera, en un estudio elaborado por Vásquez (2020) se obtuvo que este trastorno afecta en lo personal y laboral, mermando los resultados laborales, ya sea por ausencia, problemas con compañeros e incomodidad. Los autores Gherardi, Cardoso, Teixeira, Pereira, y Reisdorfer (2015) menciona que, los más susceptibles a padecer este trastorno, son aquellos que trabajan directamente con personas que requieren ayuda. Siendo los más afectados los profesionales de la salud, quienes experimentan emociones de dolor, miedo y conocen la intimidad de los pacientes. Así mismo las jornadas extensas, relaciones laborales quebrantadas, entre otros, constituyen algunos factores que colaboran a la aparición de la depresión.

Considerando a los autores Patricio Benavidez (2017) y Egziabher \& Edwards (2013), podemos decir que la depresión es uno de los factores que afecta a la población desempleada durante el SARS-CoV2, entendiendo por depresión a un estado de melancolía, tristeza e infelicidad, donde hay pérdida del estado de ánimo, decaimiento e impotencia y según De \& Gender (2013), no existe diferencia de género y no obstante, la colectividad de las personas puede sentirse así en ciertos momentos.

Gil García (2016) menciona que en Europa el costo relacionado con la depresión en el trabajo es de 617.000 millones de euros anuales, dentro de lo cual está incluido el costo del absentismo laboral, la pérdida de productividad, los gastos en salud y las indemnizaciones por discapacidad.

Wang et al, (2020) realizó un estudio en China con 1210 personas, durante la fase inicial de la pandemia del Sars-Cov-2, el cual reveló que el $13,8 \%$ de las personas mostró síntomas depresivos leves; el 12,2\%, síntomas moderados; y el 4,3\%, síntomas graves. De la misma manera se pudo observar que los varones presentaban mayores niveles de depresión, así como también las personas sin educación. 
De igual manera se reportaron niveles menores de depresión en aquellas personas que fueron informadas del aumento de pacientes recuperados y también en personas que hicieron caso a las recomendaciones de salud pública.

Ozamiz et al (2020) reseña que las personas que manifiestan tener enfermedades crónicas revelan padecer en gran medida estrés, ansiedad y depresión, en comparación con las personas que no refieren tener ninguna enfermedad crónica. Los mismos autores aluden a que el bienestar psicológico es importante al momento de afrontar el COVID-19, advertir enfermedades mentales y encargarse de las emociones, lo que implica que las personas deben prepararse psicológicamente y distinguir seguridad ante las posibles circunstancias adversas que se tienen que vivir, esto implica prevenir $y$ enfrentar la crisis gestionando medidas socio sanitarias efectivas.

\section{METODOLOGÍA}

En la presente investigación se utilizó el método descriptivo, puesto que a través de esta se identificaron cuáles fueron los niveles de depresión que tuvieron las poblaciones empleadas $y$ desempleadas, durante la crisis sanitaria Del SARS-CoV-2. Como método empírico se utilizó el "inventario de depresión de Beck" al ser uno de los representantes de las explicaciones cognitivas de la depresión, el cual fue validado en 1978. Éste es un instrumento autoadministrado de 21 ítems, que mide la severidad de la depresión en adultos y adolescentes mayores de 13 años. Cada ítem se califica en una escala de 4 puntos que va desde el 0 hasta el 3 , al final se suman dando un total que puede estar entre 0 y 63. (Beltrán, Freyre, \& Hernández-Guzmán, 2012),

Esta investigación se fundamenta en un estudio de campo realizado de manera virtual en la Ciudad de Manta, Manabí, provincia del Ecuador para lo cual se consideró una muestra total de 74 encuestas realizadas, siendo 37 empleados, trabajadores de la agencia nacional de tránsito de la Ciudad de Manta y 37 desempleados, personas residentes también en la misma Ciudad. 
Como criterio de inclusión se consideró a las personas empleadas en un rango de 18 a 60 años, residentes en la ciudad de Manta y con trabajo remunerado mínimo 3 meses. Con relación a las personas desempleadas se consideró que un rango de 18 a 60 años, residentes en la ciudad de Manta, que estén sin trabajo mínimo 3 meses y no recibieran remuneración alguna.

El Análisis de la información a través del software estadístico SPSS (Statistical Package for the Social Science), el cual es un recurso que entre sus herramientas cuenta con el análisis descriptivo, el cual es un modelo estadístico que se utiliza para identificar y representar la interacción entre variables de un conjunto, es decir muestra la existencia de variables que al relacionarse miden el mismo fenómeno. Según el autor autor Rendón-Macías, Villasís-Keever, \& Miranda-Novales (2016), el objetivo del análisis descriptivo se basa en mostrar de forma resumida los datos obtenidos en una investigación, ya sea mediante tablas, gráficos, figuras o cuadros; cada uno de ellos con una función en especial. De esta manera, los resultados alcanzados se pueden visualizar de forma clara y sencilla.

\section{RESULTADOS}

Al aplicar el análisis descriptivo se realizó el procedimiento de tabla cruzada para obtener un panorama detallado de los resultados y así validar de manera satisfactoria el estudio. En consecuencia, el autor Herrera Aráuz (2017) indica que la tabulación cruzada permite ver los datos obtenidos de manera desglosada y en forma de tablas con sus respectivas filas y columnas, para así poder analizar la correlación que existe entre dos o más variables.

De esta manera, en la comparativa del nivel de depresión y condición de empleo, se obtuvo como resultado que el $73 \%$ de los encuestados generales presenta altibajos considerados normales, donde la mayor cantidad del porcentaje la ocupan los participantes que se encuentran desempleados $(39,19 \%)$ y el menor porcentaje lo ocupan los participantes que se encuentran empleados $(33,78 \%)$, lo que es indicador de que en tiempos de pandemia las personas que presentan algún grado de depresión son las empleadas, ya que mayoría 
de los desempleados presentan altibajos considerados normales, sin embargo, en tiempos de la pandemia del COVID-19 no hay una diferencia significativa entre los porcentajes de una u otra población, ambas condiciones pueden padecer depresión en distintos grados, esto se debe principalmente a que durante esta crisis sanitaria hay en la población preocupación de perder su empleo, de no conseguir empleo, de no poder adecuarse a las nuevas modalidades de trabajo, principalmente a la virtual, miedo a la muerte y temor al contagio y a contagiar a sus allegados; el 16,2\% presenta leve perturbación del estado del ánimo, dentro de los cuales la mayoría son personas empleadas $(9,46 \%)$ y la minoría son personas desempleadas $(6,76 \%)$, lo que es indicador de que la población empleada presenta más niveles leves de depresión, lo que quiere decir que los síntomas afectan poco a la vida diaria o lo hacen solo en algún aspecto específico de la vida cotidiana; el 6,8\% presenta depresión moderada, donde el mayor porcentaje corresponde a personas desempleadas $(4,05 \%$ ) y el menor porcentaje lo ocupan las personas empleadas $(2,70 \%)$, lo que indica que la depresión moderada es más alta en personas desempleadas, lo que se refiere a que los síntomas y las limitaciones no son ni tan específicos como en la leve, ni tan generalizados como en la grave, pero en este nivel de depresión si se comienzan a manifestar consecuencias debido al estado de ánimo de las personas y su funcionalidad en cualquiera de las esferas, laboral, familiar o social; por último el 4,1\% presenta estados de depresión intermitente, esto representado sólo por la población empleada $(4,05 \%)$, lo que es indicador de que sólo los empleados presentan este grado de depresión, lo que indica que los episodios depresivos duran entre 2 y 14 días, siendo lo habitual que duren entre 5 y 7 días, además estos episodios se dan entre unas 6 y 12 veces al año, durante este tiempo las personas ven limitadas sus capacidades, ya que no sienten las ganas o el ánimo para poder desarrollar de manera normal sus actividades cotidianas y por lo tanto baja la funcionalidad de ellos principalmente en el trabajo. 
De los porcentajes obtenidos, se observa que el grado leve de depresión es el que más resalta entre todos los niveles, además los valores en general se diferencian por dos o tres puntos entre el mayor y el menor resultado mostrado por nivel de depresión, no muestra una diferencia relevante entre cada uno de los resultados, siendo una estadística pareja en cuanto a los datos recolectados. Por lo tanto, de los porcentajes obtenidos, se observa que el nivel que más porcentaje obtuvo fue el de altibajos considerados como normales, siendo 54 participantes de 74 los que se inclinaron hacia ese nivel de depresión, lo cual indica que estas personas sufren pequeños episodios de depresión que son transitorios y que no afectan la funcionalidad de la persona en sus quehaceres cotidianos ni en ningunas de las esferas que rodean al ser humano, es decir familiar, social ni laboral. Ver Figura 1.

Figura 1. Gráfico de nivel de depresión/condición de empleo

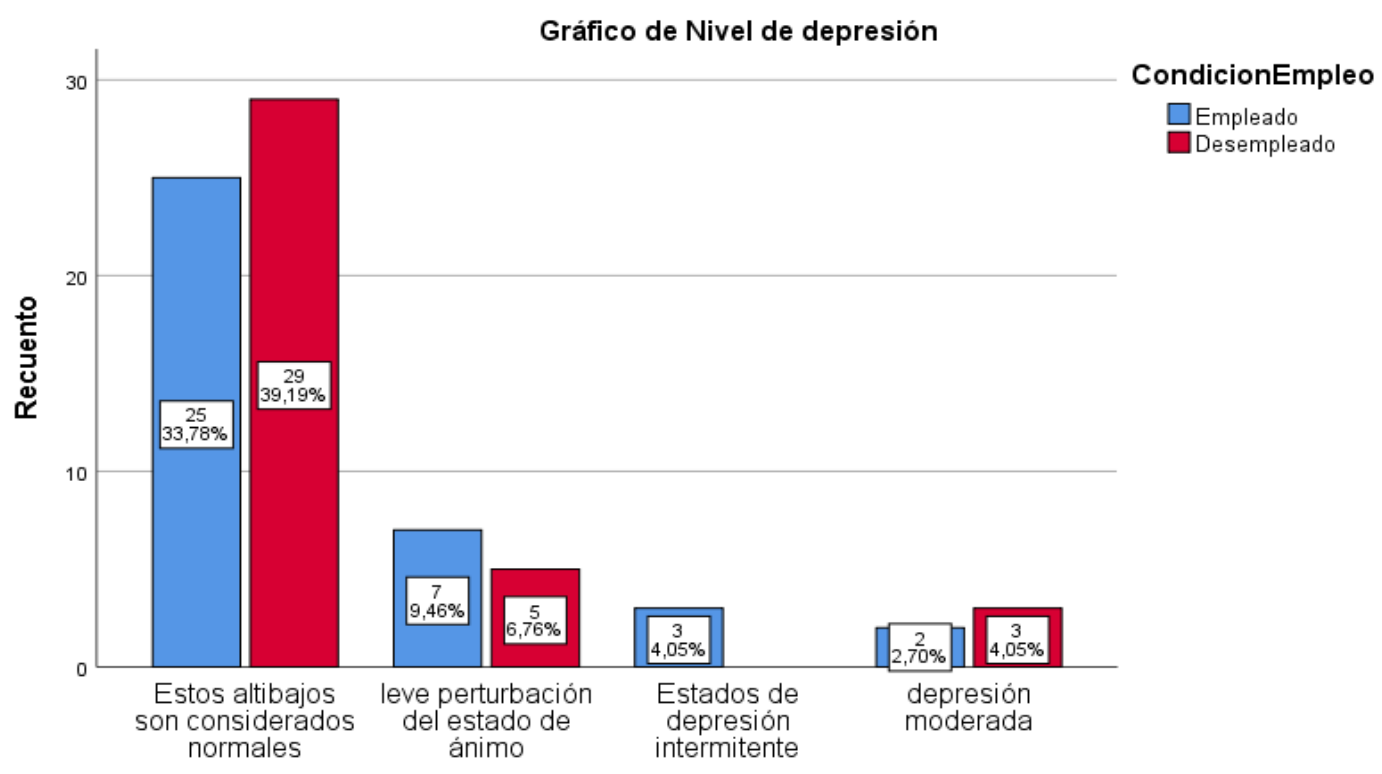

Nota. Fuente: Elaboración propia (2021).

\section{DISCUSIÓN}

Considerando al autor GherardiDonato, Cardoso, Teixeira, Pereira, \& Reisdorfer (2015) y su estudio, que, al analizar los niveles de depresión con relación a la situación laboral, se obtuvo que las personas empleadas también sufren de episodios depresivos, ya sea por estrés laboral, horarios de trabajo 
extendidos, temor al despido, entre otros factores que generan un ambiente de estrés, lo que desencadena en las perturbaciones del estado del ánimo, el cual es el nivel en donde se evidenció que las personas empleadas superan en número a las desempleadas. Así mismo, en el nivel de depresión moderada, los individuos que se encuentran desempleados superaron en porcentaje a quienes se encuentran empleados. Siendo el mismo desempleo la principal causa de depresión en estos.

Los autores Lozano Chaguay et al., (2020) manifiestan que la pandemia ha dejado un gran número de personas desempleadas provocándoles grandes problemas de depresión por las responsabilidades económicas que deben cumplir, pero que, ya no podrán asumir. Quienes mantienen el empleo, según Huarcaya-Victoria (2020) pueden generar estrés laboral, debido a la reducción de personal, teniendo que suplir las responsabilidades laborales de los compañeros que ya no se encuentran trabajando. Muchas veces este estrés laboral genera como consecuencia, entre otras a padecer de depresión, por no tener factores protectores, que le ayuden a buscar otras posibles soluciones, según Jacome (2020). Desde otra perspectiva, un estudio ejecutado en Chile por Mejía et.al., (2019) demostró que los trabajadores asalariados sometidos a factores estresantes laborales tienen una mayor posibilidad de padecer síntomas de trastornos mentales, entre ellos la depresión.

Según Torres López, MunguíaCortés, \& Torres-Valdovinos (2018), mencionan que el estar desempleado implica un factor de riesgo para los problemas 0 trastornos mentales, mientras que al estar empleado o una reincorporación al trabajo sugieren efectos protectores para la salud mental. No obstante, un ambiente laboral adverso, como el que se está experimentando en la actualidad por la crisis sanitaria, el teletrabajo, problemas de mobbing, malas relaciones sociales, consumo de sustancias, absentismo laboral y pérdidas en la productividad, pueden ocasionar problemas físicos y psíquicos a los empleados de 
cualquier sector laboral, considerando como referencia a Vera et al., (2018).

En los cuatro niveles de depresión planteados en la investigación, se determinó que en el primero se obtuvo la mayor cantidad de respuestas con el $73 \%$ global de los datos obtenidos. Esto se debe a que existen situaciones en las cuales los altibajos emocionales afectan a cualquier tipo de persona sin importarle su condición social y económica, y son momentos que suelen desaparecer de la misma forma en la que se presentan, son fugaces. En el segundo nivel se obtuvo que el $16.2 \%$ de los participantes presentan una leve perturbación del estado de ánimo, esto se atribuye a diversos factores, ya sean problemas familiares, personales, laborales. Este tipo de situaciones se los puede controlar con buena compañía de personas allegadas a quienes sufren de esta perturbación, lo cual colabora de manera positiva a mejorar el estado de ánimo y brindarle las fuerzas que requieren para confrontar la situación. En el tercer nivel se obtuvo que un $4.1 \%$ de los participantes sufren de estados de depresión intermitente, en donde en un momento se encuentran bien y al siguiente sienten una baja del estado de ánimo, lo cual viene siendo como un bucle repetitivo de experimentación de emociones entre agradables y desagradables. En el último nivel se obtuvo que el $6.8 \%$ de los encuestados sufre de depresión moderada y en su mayoria son aquellas personas que se encuentran desempleadas, por lo que la ausencia de empleo es un factor determinante en el desarrollo de un trastorno depresivo.

Por lo tanto, podemos observar que en el tercer nivel se obtuvo que tres personas se encontraban ubicadas en esta posición, y en su totalidad (3) eran aquellos que contaban con un empleo. Finalmente, en el último nivel se muestra que 5 personas están ubicadas en este grado, de las cuales 3 son desempleados y los 2 restantes cuenta con un empleo. Considerando estos resultados y con la aparición del Covid 19 los niveles de estrés han ido en aumento en el ser humano, sea que labore o no, los momentos en los cuales el ánimo no está en su punto máximo afecta a cualquier persona sin distinción laboral alguna, lo que conlleva a que 
la depresión es una enfermedad que ataca a cualquiera que haya pasado o esté pasando una situación complicada para la cual sienten que no hay vías de solución alguna, es por esto que se hace muy importante que las personas que han salido con resultados alterados y la población en general, cuando comience a sentir síntomas, acuda a especialistas de la salud mental para evitar que sus cuadros se agraven o se puedan desencadenar trastornos mentales más complejos.

En consecuencia, en todos los niveles detallados, se observa que las personas que cuentan con un empleo no se diferencian en nada a las personas desempleadas, debido a que los resultados obtenidos evidencian que un buen porcentaje de personas que cuentan con un trabajo estable sufren de depresión, ya sea altibajos emocionales, perturbaciones del estado de ánimo, e incluso una depresión intermitente que se puede llegar a convertir en una depresión moderada dependiendo de la situación en la cual se desenvuelva el individuo. $Y$ en la actualidad considerando Lozano Chaguay et al., (2020), con la aparición del covid 19 esta enfermedad se ha visto en un aumento progresivo y mucho más frecuente, e incluso durante la pandemia se ha incremendado, afectando a cualquier persona que no cuente con el acompañamiento y la atención necesaria para afrontar este trastorno, ya que esto requiere de mucho apoyo.

\section{CONCLUSIONES}

Al término de esta investigación y habiendo obtenido los resultados del estudio de campo aplicado a 74 personas con condiciones de empleos distintas (empleado y desempleado), se muestra que la depresión está presente en cualquiera persona, sea cual sea su condición laboral. Considerando el primer nivel de depresión se obtuvo que 54 personas consideraron que se encuentran en ese rango, en donde 29 eran desempleados y 25 contaban con un empleo. Así mismo, en el segundo nivel, un total de 12 personas se encuentran ubicadas en esta situación, distribuidas en 7 persona con empleo y 5 desempleadas. 
Todas estas situaciones requieren de apoyo, en unas solamente con el acompañamiento de personas que brinden paz, seguridad y confianza. $Y$ en otras situaciones se requiere de ayuda profesional, debido a que las crisis depresivas afectan el estado mental y emocional de quien lo padece. En estos cuatro niveles planteados se observa situaciones que los encuestados consideran normales, como los altibajos emocionales, y el cambio en el estado de ánimo, considerando Beltrán et al., (2012). Pero también existen situaciones que, de volverse recurrentes se necesitaría ayuda profesional para llevar de mejor manera la enfermedad.

Por lo que se concluye que este estudio fue factible aplicando el análisis descriptivo a los datos obtenidos del inventario de depresión de Beck y determinándose que los niveles de estrés han hecho vulnerable a las personas empleadas por sobre las personas desempleadas a desarrollar una depresión.

\section{BIBLIOGRAFÍA}

Balluerka, N., Gómez, J., Hidalgo M., Gorostiaga, A., Espada, J.,
Padilla J., Santed M. (2020). Las consecuencias psicológicas de la covid-19 y el confinamiento. Servicio de Publicaciones de la Universidad del País Vasco Euskal Herriko Unibertsitateko Argitalpen Zerbitzua.

https://www.ub.edu/web/ub/c a/menu_eines/noticies/docs/ Consecuencias_psicologicas _COVID-19.pdf

Beltrán, M. del C., Freyre, M. Á., \& Hernández-Guzmán, (2012). The beck Depression Inventory: Its validity in adolescent population. Terapia Psicologica, 30(1), 513.

https://doi.org/10.4067/s071848082012000100001

Benavides, P. (2017). La depresión, evolución del concepto desde la melancolía hasta la depresión como enfermedad física. Revista puce.105, 171188.

http://www.revistapuce.edu.e c/index.php/revpuce/article/vi ew/119/149

Castellón Leal, E., Ibern Regàs, P., Gili Planas, M., Lahera Forteza, G., Sanz González, J., \& Saiz Ruiz, J. (2016). Management of depression in the work setting: Key recommendations. Psiquiatria Biologica, 23(3), 112-117. https://doi.org/10.1016/j.psiq. 2016.08.003 
CEPAL (Comisión Económica para América Latina y el Caribe) (2020), "América Latina y el Caribe ante la pandemia del COVID-19: efectos económicos y sociales", Informe Especial, $\mathrm{N}^{\circ} 1$, Santiago

De, D. D., \& Gender, D. (2013). Depresión. Diferencias de género, 17(3), 193-217.

Domínguez, R., Zelaya, S., Gutiérrez, M., \& Castellanos, E. (2020). Recomendable Medidas de protección en personal de salud para disminución de riesgo de contagio de COVID-19. Instituto Nacional de Salud, 117. Retrieved from https://docs.bvsalud.org/biblio ref/2020/08/1087768/medida s-de-proteccion-debioseguridad-p-de-salud17ago20.pdf

Egziabher, T. B. G., \& Edwards, S. (2013). Depresion un problema de salud. Africa's Potential for the Ecological Intensification of Agriculture, 53(9), 1689-1699.

Garcia, W. (2017). CURSO DE EXPERTO: "METODOLOGÍA DE LA INVESTIGACIÓN ." CURSO DE EXPERTO: “ METODOLOGÍA DE LA INVESTIGACIÓN ," 1, 23.

Gherardi-Donato, E. C. da S., Cardoso, L., Teixeira, C. A. B., Pereira, S. de S., \&
Reisdorfer, E. (2015). Association between depression and work stress in nursing professionals with technical education level. Revista Latino-Americana de Enfermagem, 23(4), 733-740. https://doi.org/10.1590/01041169.0069 .2610

Gil García, D. (2016). Los costes económicos de la depresión en el trabajo.

Hefetz, A., \& Liberman, G. (2017). The factor analysis procedure for exploration: a short guide with examples / El análisis factorial exploratorio: una guía breve con ejemplos. Taylor \& Francis, 29(3), 526-562. https://doi.org/10.1080/11356 405.2017.1365425

Hernández, j. (2020). Impacto de la COVID-19 sobre la salud mental de las personas. Revista científica Villa Clara $24 \quad$ (3), 578-594. http://scielo.sld.cu/pdf/mdc/v2 4n3/1029-3043-mdc-24-03578.pdf

Herrera Aráuz, D. (2017). Estadística con SPSS.

Huarcaya-Victoria, J. (2020). Consideraciones sobre la salud mental en la pandemia de COVID-19. Revista Peruana de Medicina Experimental y Salud Pública, 37(2), 327-334. https://doi.org/10.17843/rpme sp.2020.370.5419 
Jácome, A. (2020). Salud Mental Y Trabajo: Estudio De Depresión Y Ansiedad Ante EI Covid-19 En El Personal De Cajas De Un Hospital Privado De Quito-Ecuador 2020. Repositorio Digital Universidad Internacional SEK, 1-77. Retrieved from http://repositorio.uisek.edu.ec /handle/123456789/3857

Lozano Chaguay, S., Robledo Galeas, R., \& Lozano Chaguay, L. A. (2020). Desempleo en tiempos de covid-19: efectos socioeconómicos en el entorno familiar. Journal of Science and Research: Revista Ciencia e Investigación, 5(4), 187-197.

Macavilca-cruz, M., Wuhan, E., \& Wuhan, D. (2021). Estrategias de control de la COVID-19 en unidades de hospitalización psiquiátrica ., 84(1), 69-71.

Maldonado Luna, S. (2007). Manual Práctico Para El Diseño De La Escala Likert. Revista Xihmai, 2(4), 6-8.

Martínez Sierra, P. (2020). Aproximación a las implicaciones sociales de la pandemia del COVID19 en niñas, niños y ado lescentes: el caso de México. Sociedad E Infancias, 4,255-258. https://dialnet.unirioja.es/servl et/articulo?codigo $=7517714$.

Mejia, C. R., Chacon, J. I., Enamorado, O. M., Garnica,
L. R., Chacón, S. A., \& García, Y. A. (2019). Factores asociados al estrés laboral en trabajadores de seis países de Latinoamérica. Rev. Asoc. Esp. Espec. Med. Trab, 28(3), 204-211. Retrieved from http://scielo.isciii.es/pdf/medtr a/v28n3/1132-6255-medtra28-03-204.pdf

Morales, C. (2017). La depresión: Un reto para toda la sociedad del que debemos hablar. Revista Cubana de Salud Pública, 43(2), 136-138. Retrieved from http://scielo.sld.cu

Organización Mundial de la Salud. (2017). Día Mundial de la Salud Mental 2017 - La salud mental en el lugar de trabajo. https://www.who.int/mental_h ealth/es/\#: :text=M\%C3\%A1s $\% 20$ de $\% 20300 \% 20$ millones \%20de,en\%20el\%20lugar\%2 0de\%20trabajo

Organización Mundial de la Salud. (2019).

Coronavirus. https://www.who.int/es/healthtopics/coronavirus\#tab=tab_1

Organización Internacional del Trabajo. (2020). EI COVID-19 $y$ el mundo del trabajo: Repercusiones y respuestas. Observatorio de la OIT. $1^{\text {a }}$ edición (1), 2 - 18.

Ojeda-Casares, H., \& Gerardo de Cosio, F. (2020). COVID-19 y salud mental: mensajes clave. Ops-Oms, (1), 1-7. Retrieved from

https://www.paho.org/venezu 
ela/index.php?option=com_d ocman\&view=download\&slug =covid19-y-salud-mentalmensajes-clave\&Itemid=466

Ozamiz, N., Dosil, M., Picaza, M., Idoiaga, N. (2020). Niveles de estrés, ansiedad y depresión en la primera fase del brote del COVID-19 en una muestra recogida en el norte de España. Cadernos de saúde pública, 36 (4), 2-10. https://doi.org/10.1590/0102311 X00054020

Patricio Benavidez. (2017). Hasta La Depresión Como Depression, Evolution of the Concept. Revista Puce, 8, 173-188.

Rendón-Macías, M. E., VillasísKeever, M. Á., \& MirandaNovales, M. G. (2016). Estadística descriptiva. Revista Alergia México, 63(4), 397.

https://doi.org/10.29262/ram.v $63 \mathrm{i} 4.230$

Romero Díaz, T., \& Martínez Gimeno, A. (2017). Elaboration and validation of an evaluation questionnaire to teacher performance through Exploratory Factor Analysis. Revista Científica de FAREMEstelí. Medio Ambiente, Tecnología y Desarrollo Humano, 6(22), 18-30.

Santos, C., \& León, A. (2019). LA $\begin{array}{lrr}\text { MEDICIÓN } & \text { DE } & \text { LOS } \\ \text { RESULTADOS } & \text { SOCIALES } \\ \text { EN } \quad \text { EL } & \text { SECTOR }\end{array}$
BANANERO; UN ESTUDIO DE CORTE TRANSVERSAL EN LA PROVINCIA DE LOS RÍOS, REPÚBLICA DEL ECUADOR. Universidad, Ciencia y Tecnología, 23, 107-118.

Serrano Rodríguez, R., Amor Almedina, M. I., Guzman Cedeño, Á., \& GuerreroCasado, J. (2018). Validation of an Instrument to Evaluate the Development of University Teaching Competences in Ecuador. Journal of Hispanic Higher Education. https://doi.org/10.1177/15381 92718765076

Torres López, T. M., MunguíaCortés, J. A., \& TorresValdovinos, M. M. (2018). Representaciones sociales de empleo y desempleo en estudiantes universitarios de Quito, Ecuador. Revista Reflexiones, 97(2), 7. https://doi.org/10.15517/rr.v9 7i2.32704

Vasques Elera, L. (2020). Ansiedad, depresión y estrés en trabajadores del Hospital Cayetano Heredia durante la pandemia de COVID 19 durante el año 2020. https://repositorio.ucv.edu.pe/ bitstream/handle/20.500.1269 2/49971/Vasquez_ELESD.pdf?sequence $=1$ \&isAllow $\mathrm{ed}=\mathrm{y}$ 
Vera, M., Vélez, C. A., \& Córdova, M. F. (2018). Efectos de la Depresión en el Bienestar Laboral: El Rol Modulador del Apoyo Social en el Trabajo. Investigatio, (10), 1-9. https://doi.org/10.31095/inves tigatio.2018.10.1

Wang, C., Pan, R., Wan, X., Tan, Y., $\mathrm{Xu}$, L., S.Ho, C., C.Ho, R. (2020). Immediate Psychological Responses and Associated Factors during the Initial Stage of the 2019 Coronavirus Disease (COVID19) Epidemic among the General Population in China. Int. J. Environ. Res. Public Health. $17 \quad$ (5), 2-25. https://doi.org/10.3390/ijerph1 7051729 